Bull. Austral. Math. Soc.

VOL. 37 (1988) [429-435]

\title{
SHARP CONDITIONS FOR OSCILLATIONS CAUSED BY RETARDED AND ADVANCED PERTURBATIONS
}

\author{
J.E. BowCOCK AND Y.H. YU
}

In this paper we study the oscillatory behaviour of solutions of equations of the forms $(A) y^{\prime}(t)+p y(t)+\sum_{i=1}^{n} p_{i} y\left(t-\tau_{i}\right)=0$ and $(B) y^{\prime}(t)-p y(t)-\sum_{i=1}^{n} p_{i}\left(y\left(t+\tau_{i}\right)\right)=0$, where $p_{i}$ and $\tau_{i}$, for $i=1,2, \ldots, n$, are positive constants and $p$ is an arbitary constant. Three necessary and sufficient conditions for all solutions of $(A)$ and $(B)$ respectively to be oscillatory are established. These results extend and generalise previous results in a new approach.

\section{INTRODUCTION}

Recently the study of the oscillatory behaviour of solutions of first order differential equations with retarded and advanced perturbations has been a subject of great interest. A characteristic feature of the work is that when the perturbation vanishes the resulting first order ordinary differential equation has no oscillatory behaviour. In the literature [1-6], the work in this direction is centred around functional differential equations in which the rate functions depend only on the past history or the future of a state of a system. However, in general, the rate functions not only depend on the past history or future state of a system, but also on its present state.

In the present work, we give three necessary and sufficient conditions for all solutions of $(A)$ and $(B)$ to be oscillatory. Our results are based on a very recent and interesting result of Arino, Györi and Jawhari [1]. Furthermore, our results extend and generalise the results of $[1-6]$ in a new approach.

Lemma 1.1. [1] Consider a linear scalar functional differential equation

$$
x^{\prime}(t)=l\left(x_{t}\right)
$$

Then all the solutions of $(L)$ are oscillatory if and only if the characteristic equation $-\lambda=l\left(e^{-\lambda}\right)$ of $(L)$ has no real root.

Received 18 August, 1987

The Second Author would like to express his gratitude to Professor W.N. Everitt for his enthusiastic help. This research was partially supported by the British Council.

Copyright Clearance Centre, Inc. Serial-fee code: 0004-9729/88 \$A2.00+0.00. 
2. DiffERENTIAL EQUATIONS WITH SEVERAL RETARDED PERTURBations

Consider the differential equation with retarded perturbations

$$
y^{\prime}(t)+p y(t)+\sum_{i=1}^{n} p_{i} y\left(t-\tau_{i}\right)=0
$$

where $p$ is an arbitary constant and $p_{i}$ and $\tau_{i}$, for $i=1,2, \ldots, n$, are positive constants.

The characteristic equation of $(2.1)$ is

$$
-\lambda+p+\sum_{i=1}^{n} p_{i} \exp \left(\lambda \tau_{i}\right)=0
$$

Lemma 2.1. Put

$$
\left\{\begin{array}{l}
\lambda_{0}=p \\
\lambda_{k}=p+\sum_{i=1}^{n} p_{i} \exp \left(\lambda_{k-1} \tau_{i}\right) \quad k=1,2, \ldots
\end{array}\right.
$$

Then $\left\{\lambda_{k}\right\}_{k=0}^{\infty}$ is a monotonically increasing sequence.

Proof: From (2.3) we have

$$
\lambda_{1}-\lambda_{0}>\mathbf{0}
$$

therefore, by induction,

$$
\lambda_{k+1}-\lambda_{k}=\sum_{i=1}^{n} p_{i} \exp \left(\lambda_{k-1} \tau_{i}\right)\left[\exp \left(\lambda_{k}-\lambda_{k-1}\right) \tau_{i}-1\right]>0, \quad k=1,2, \ldots
$$

The proof of the Lemma is complete.

TheOREM 2.2. A necessary and sufficient condition for all solutions of (2.1) to be oscillatory is that

$$
\lim _{k \rightarrow \infty} \lambda_{k}=+\infty
$$

ProOf: Assume first that (2.4) does not hold. From Lemma 2.1 the sequence $\left\{\lambda_{k}\right\}_{k=0}^{\infty}$ converges. In view of (2.3), there exists a real root of (2.2). Then (2.1) has a non-oscillatory solution.

Assume conversely, that not all solutions of (2.1) oscillate. From Lemma 1.1 we know that there exists a real number $\lambda^{\star}$ such that

$$
-\lambda^{\star}+p+\sum_{i=1}^{n} p_{i} \exp \left(\lambda^{\star} \tau_{i}\right)=0
$$


From (2.5) and (2.3) we have

$$
\lambda^{\star}-\lambda_{0}>0
$$

Therefore, by induction,

$$
\lambda^{\star}-\lambda_{k}=\sum_{i=1}^{n} p_{i} \exp \left(\lambda_{k-i} \tau_{1}\right)\left[\exp \left(\lambda^{\star}-\lambda_{k-1}\right) \tau_{i}-1\right]>0, \quad k=1,2, \ldots
$$

(2.6) and (2.4) lead to a contradiction. The proof of the Theorem is complete.

THEOREM 2.3. A necessary and sufficient condition for all solutions of (2.1) to be oscillatory is that

$$
-\xi+\sum_{i=1}^{n} p_{i} \exp (p+\xi) \tau_{i}>0, \quad \xi>0
$$

Proof: Assume first that (2.7) does not hold. We may then choose $\xi^{\star}>0$, such that

$$
-\xi^{\star}+\sum_{i=1}^{n} p_{i} \exp \left(p+\xi^{\star}\right) \tau_{i}=\mathbf{0}
$$

Set

$$
\lambda^{\star}=\xi^{\star}+p
$$

then (2.8) can be rewritten as (2.5). Therefore, (2.1) has a non-oscillatory solution.

Conversely, assume that not all solutions of (2.1) oscillate. From Theorem 2.2 we know that (2.4) does not hold. In view of Lemma 2.1 the sequence $\left\{\lambda_{k}\right\}_{k=0}^{\infty}$ converges.

Set

$$
\lambda_{k}=\xi_{k}+p, \quad k=0,1,2, \ldots
$$

Then

$$
\left\{\begin{array}{l}
\xi_{0}=0 \\
\xi_{k}=\sum_{i=1}^{n} p_{i} \exp \left(p+\xi_{k-1}\right) \tau_{i}, \quad k=1,2, \ldots .
\end{array}\right.
$$

Therefore, the sequence $\left\{\xi_{k}\right\}_{k=0}^{\infty}$ converges too, and we have

$$
\lim _{k \rightarrow \infty} \xi_{k}=\xi^{\star} \text {. }
$$

From (2.9) we know that $\xi_{k}>0$, for $k=1,2, \ldots$, and the sequence $\left\{\xi_{k}\right\}_{k=0}^{\infty}$ is monotonically increasing. Hence we have $\xi^{\star}>0$. From (2.9) and (2.10) we get

$$
-\xi^{\star}+\sum_{i=1}^{n} p_{i} \exp \left(p+\xi^{\star}\right) \tau_{i}=0, \quad \xi^{\star}>0 .
$$

(2.11) and (2.7) lead to a contradiction. The proof of the Theorem is now complete. 
Remark 1. Theorem 2.3 generalises the main results of $[6,4]$ and Proposition 1 of [1].

THEOREM 2.4. A necessary and sufficient condition for all solutions of (2.1) to be oscillatory is that

$$
\sum_{i=1}^{n} p_{i} \tau_{i} \exp \left(p \tau_{i}\right)>e^{-1}
$$

Proof: Assume first that all solutions of (2.1) oscillate. From Theorem 2.3, (2.7) holds, or, equivalently,

$$
\sum_{i=1}^{n} p_{i} \xi^{-1} \exp (p+\xi) \tau_{i}>1, \quad \xi>0
$$

Each $p_{i} \xi^{-1} \exp (p+\xi) \tau_{i}$ is minimised by setting $\xi=\tau_{i}^{-1}$ and thus, has its minimum equal to $p_{i} \tau_{i} \exp \left(p \tau_{i}+1\right)$. So if (2.13) holds, we have

$$
\sum_{i=1}^{n} p_{i} \tau_{i} \exp \left(p \tau_{i}+1\right)>1
$$

which implies (2.12).

Conversely, if (2.12) holds, from (2.13) and (2.14), we know that (2.7) is true. In view of Theorem $2.3,(2.1)$ has only oscillatory solutions. The proof of the Theorem is complete.

Remark 2. Theorem 2.4 extends and generalises the corresponding results of $[2,3$, 5] and Proposition 2 of [1].

\section{Differential EqUations WITh SEVERAL adVANCED PERTURBations}

Consider the differential equation with advanced pertrubations

$$
y^{\prime}(t)-p y(t)-\sum_{i=1}^{n} p_{i} y\left(t+\tau_{i}\right)=0
$$

where $p$ is an arbitary constant and, $p_{i}$ and $\tau_{i}$, with $i=1,2, \ldots, n$, are positive constants.

The characteristic equation of $(3.1)$ is

$$
\lambda+p+\sum_{i=1}^{n} p_{i} \exp \left(-\lambda \tau_{i}\right)=0 .
$$


Lemma 3.1. Put

$$
\left\{\begin{array}{l}
\lambda_{0}=-p \\
\lambda_{k}=-p-\sum_{i=1}^{n} p_{i} \exp \left(-\lambda_{k-1} \tau_{i}\right), \quad k=1,2, \ldots
\end{array}\right.
$$

Then $\left\{\lambda_{k}\right\}_{l=0}^{\infty}$ is a monotonically decreasing sequence.

Proof: From (3.3) we have

$$
\lambda_{1}-\lambda_{0}<0
$$

therefore, by induction,

$$
\lambda_{k+1}-\lambda_{k}=\sum_{i=1}^{n} p_{i} \exp \left(-\lambda_{k} \tau_{i}\right)\left[\exp \left(\lambda_{k}-\lambda_{k-1}\right) \tau_{i}-1\right]<0, \quad k=1,2, \ldots
$$

The proof of the Lemma is complete.

Theorem 3.2. A necessary and sufficient condition for all solutions of (3.1) to be oscillatory is that

$$
\lim _{k \rightarrow \infty} \lambda_{k}=-\infty
$$

Proof: Assume first that (3.4) does not hold. From Lemma 3.1 the sequence $\left\{\lambda_{k}\right\}_{k=0}^{\infty}$ converges. In view of (3.3), there exists a real root of (3.2). Then (3.1) has a non-oscillatroy solution.

Assume conversely, that not all solutions of (3.1) oscillate. From Lemma 1.1 we know that there exists a real $\lambda^{\star}$ such that

$$
\lambda^{\star}+p+\sum_{i=1}^{n} p_{i} \exp \left(-\lambda^{\star} \tau_{i}\right)=0
$$

From (3.5) and (3.3) we have

$$
\lambda^{\star}-\lambda_{0}<0
$$

Therefore,

$$
\lambda^{\star}-\lambda_{k}=\sum_{i=1}^{n} p_{i} \exp \left(-\lambda^{\star} \tau_{i}\right)\left[\exp \left(\lambda^{\star}-\lambda_{k-1}\right) \tau_{i}-1\right]<0, \quad k=1,2, \ldots
$$

The inequalities (3.6) contradict (3.4), and the proof of the Theorem is complete. 
Theorem 3.3. A necessary and sufficient condition for all solutions of (3.1) to be oscillatory is that

$$
\xi+\sum_{i=1}^{n} p_{i} \exp (p-\xi) \tau_{i}>0, \quad \xi<0
$$

PROOF: Assume first that (3.7) does not hold. Then we may choose $\xi^{\star}<0$, such that

$$
\xi^{\star}+\sum_{i=1}^{n} p_{i} \exp \left(p-\xi^{\star}\right) \tau_{i}=0
$$

Set

$$
\lambda^{\star}=\xi^{\star}-p
$$

then (3.8) can be rewritten as (3.5). Therefore, (3.1) has a non-oscillatroy solution.

Conversely, assume that not all solutions of (3.1) oscillate. From Theorem 3.2 we know that (3.4) does not hold. In view of Lemma 3.1 the sequence $\left\{\lambda_{k}\right\}_{k=0}^{\infty}$ converges. Set

$$
\lambda_{k}=\xi_{k}-p, \quad k=0,1,2, \ldots
$$

then

$$
\left\{\begin{array}{l}
\xi_{0}=0 \\
\xi_{k}=-\sum_{i=1}^{n} p_{i} \exp \left(p-\xi_{k-1}\right) \tau_{i}, \quad k=1,2, \ldots
\end{array}\right.
$$

Therefore the sequence $\left\{\xi_{k}\right\}_{k=0}^{\infty}$ converges too, so we have

$$
\lim _{k \rightarrow \infty} \xi_{k}=\xi^{*}
$$

From (3.9) we know that $\xi_{k}<0$, for $k=1,2, \ldots$ and the sequence $\left\{\xi_{k}\right\}_{k=0}^{\infty}$ is monotonically decreasing. Hence we get $\xi^{*}<0$. From (3.9) and (3.10) we have

$$
\xi^{\star}+\sum_{i=1}^{n} p_{i} \exp \left(p-\xi^{\star}\right) \tau_{i}=0, \quad \xi^{\star}<0
$$

(3.11) and (3.7) lead to a contradiction. The proof of the Theorem is complete.

Remark 3. Theorem 3.3 generalises the main results of $[4,6]$ and Proposition 1 of $[1]$. 
Theorem 3.4. A necessary and sufficient condition for all solutions of (3.1) to be oscillatory is that.

$$
\sum_{i=1}^{n} p_{i} \tau_{i} \exp \left(p \tau_{i}\right)>e^{-1}
$$

Proof: Assume first that all solutions of (3.1) oscillate. From Thoerem 3.3, (3.7) holds, or equivalently,

$$
-\sum_{i=1}^{n} p_{i} \xi^{-1} \exp (p-\xi) \tau_{i}>1, \quad \xi<0
$$

Each $-p_{i} \xi^{-1} \exp (p-\xi) \tau_{i}$ is minimised by setting $\xi=-\tau_{i}^{-1}$, and thus has its minimum equal to $p_{i} \tau_{i} \exp \left(p \tau_{i}+1\right)$.

So if (3.13) holds, we have

$$
\sum_{i=1}^{n} p_{i} \tau_{i} \exp \left(p \tau_{i}+1\right)>1
$$

which implies (3.12). Conversely, if (3.12) holds, from (3.13) and (3.14) we know that (3.7) is true. In view of Theorem $3.3,(3.1)$ has only oscillatory solutions. The proof of the theorem is complete.

Remark 4. Theorem 3.4 extends and generalises theorems 3.1 and 3.2 of [5].

\section{REFERENCES}

[1] O. Arino, I Gyröri and A. Jawhari, 'Oscillation criteria indelay equations', J. Differential Equations 53 (1984), 115-123.

[2] B.R. Hunt and J.A. Yorke, 'When all solutions of $x^{\prime}=-\sum q_{i}(t) x\left(t-T_{i}(t)\right)$ oscillate', J. Differential Equations 63 (1984), 139-145.

[3] G. Ladas, 'Sharp conditions for oscillations caused by delays', Applicable Anal. 9 (1979), 93-98.

[4] G. Ladas, Y.G. Sficas and I.P. Stavroulakis, 'Necessary and sufficient conditions for oscillations', Amer. Math. Monthly 90 (1983), 637-640.

[5] G. Ladas and I.P. Stavroulakis, 'Oscillations caused by several retarded and advanced argument', J. Differential Equations 44 (1982), 134-152.

[6] M.I. Tramov, 'Conditions for oscillatory solutions of first order differential equations with delayed arguments', Izv. Vyssh. Uchebn. Zaved. Mat. 19 (3) (1975), 92-96.

Dr. J.E. Bowcock

Department of Mathematics

The University of Birmingham

P.O. Box 363

Birmingliam, B15 2TT

United Kingdom
Dr. Y.H. Yu

Institute of Applied Mathematics

Academia Sinica

Beij̈ng 100080

China 